\title{
Efficient global multidisciplinary optimization based on surrogate models
}

\author{
S. Dubreuil, N. Bartoli† T. Lefebvre* \\ ONERA/DTIS, Université de Toulouse, Toulouse, France \\ C. Gogu $\ddagger$ \\ Université de Toulouse, Toulouse, France
}

\begin{abstract}
In order to solve MultiDisciplinary Optimization problems involving computationally expensive disciplinary solvers, we propose a surrogate based approach where each disciplinary solver is replaced by an inexpensive surrogate model. As an approximation error is introduced with the use of surrogate models, a probabilistic framework is considered to manage the uncertainty propagation error from the MultiDisciplinary Analysis to the objective function of the optimization problem. This leads to the construction of an adaptive surrogate-based approach, called EGMDO for Efficient Global Multidisciplinary Optimization, to solve MDO problems. The approach is based on the adaptive enrichment of the disciplinary surrogate models in areas where the optimum is likely to be. Two applications are considered with a significant reduction in terms of number of calls to the expensive disciplinary solvers: a reduction by up to a factor of 30 is observed on the Sellar test problem and in a preliminary aircraft design test case.
\end{abstract}

\section{Introduction}

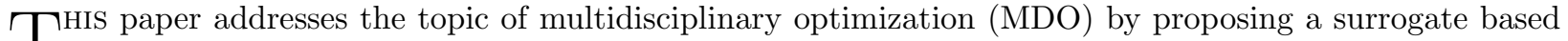
1 approach for carrying out the MDO. The method proposed focuses on multidisciplinary analysis (MDA) involving various disciplinary solvers with feedback coupling (i.e. the MDA is modeled as a non linear system of equations). Moreover, it is assumed that the gradient of the disciplinary solvers with respect to the design variables are not provided by the solvers and that the numerical cost of each solver is important (i.e. computation of gradient by finite differences is intractable).

In this context, adaptive surrogate based optimization approaches seem to be relevant and have shown efficiency in the context of optimization of monodisciplinary black-box functions (see 1-3). However, to the best of our knowledge, their are only few attempts to adapt them to the MDO context. The proposed approach, inspired by the Efficient Global Optimization (EGO) algorithm, ${ }^{1}$ can be seen as an uncoupled MDO formulation in which each disciplinary solver is approximated by a metamodel. Then, these surrogate models are adaptively enriched in promising areas of the design space with respect to the minimum value of the objective function. Obviously the difficulties lie in the research of the promising areas of the design space, given that the various disciplines are coupled, thus rendering non-trivial the choice of the disciplines to be enriched and the choice of the most relevant input values. In order to properly define these areas, the probabilistic framework is retained. Hence, each disciplinary solver is approximated by a Gaussian process over the design and coupling variable spaces. Then, by carefully monitoring the uncertainty introduced by the use of these surrogate models in the MDO process, the proposed algorithm finds relevant points of the design space where the disciplinary surrogate models should be enriched.

In order to set up this strategy, several issues must be tackled among which:

- It is assumed that each disciplinary solver is approximated by a Gaussian process. However, the MDA couples these surrogate models through a non linear system of equations. Hence, the coupling variables

* Research Engineer, Information Processing and Systems Department

$\dagger$ Research Engineer, Information Processing and Systems Department, AIAA Member

${ }^{\ddagger}$ Associate professor, Institut Clément Ader (ICA), CNRS, ISAE-SUPAERO, UPS, INSA, Mines-Albi, AIAA Member. 
are random variables with unknown probability distribution as well as the objective function (computed from the solution of the MDA). Consequently, the uncertainty propagation of the Gaussian surrogate models through the MDA to the objective function is the first step of the proposed approach.

- Due to the modelling uncertainty induced by the use of surrogate models (Gaussian processes), the objective function is a random field over the design space. The representation of this random field is a keystone of the proposed approach.

- An enrichment criterion must be defined in order to find relevant areas of the design space where the disciplinary surrogate models must be enriched.

The next section introduces the general problem we are interested in, details the three previously mentioned steps and presents the proposed uncoupled MDO method denoted by Efficient Global Multidisciplinary Optimization (EGMDO). Section III presents two applications of the proposed algorithm on the analytical Sellar problem ${ }^{4}$ and on an aircraft design test case investigated in the H2020 AGILE European project. ${ }^{5,6}$ Section IV draws conclusions and presents future developments.

\section{EGMDO description}

\section{A. Overview}

Objective of this paper is to solve an MDO problem involving $n_{d}$ disciplines as follows:

$$
\min _{x \in \mathcal{X}} y_{o b j}\left(x, y_{i}\right) i=1, \cdots, n_{d}
$$

where $y_{o b j}$ is the objective function (to minimize in this case) depending of $x$ and $y_{i}$. The design variables $x$ belongs to a design space $\mathcal{X} \subset \mathbb{R}^{n}$. The coupling variables $y_{i}, i=1, \cdots, n_{d}$ are solution of the non linear system of $n_{d}$ equations, called multidisciplinary analysis (MDA),

$$
y_{i}=f_{i}\left(x, y_{c^{(i)}}\right), i=1, \cdots, n_{d} \quad \forall x \in \mathcal{X}
$$

where $y_{c^{(i)}}$ is the vector of the coupling variables for the discipline $i$. The set of indexes denoted by $c^{(i)}$ identifies the coupling variables i.e. $\#\left(c^{(i)}\right) \leq\left(n_{d}-1\right)$ and $i \notin c^{(i)}$. Finally, $f_{i}$ is the solver of discipline $i$. Let us note that, with the previously introduced notations, disciplines $i$ and $j$ are said to have a feedback loop if $i \in c^{(j)}$ and $j \in c^{(i)}$. In the following, it is assumed that Eq. (2) contains at least one feedback coupling.

In order to illustrate such a problem, Figure 1 presents on the left a graphical representation of an MDO problem involving two disciplines and two coupling variables $y_{1}$ and $y_{2}$.
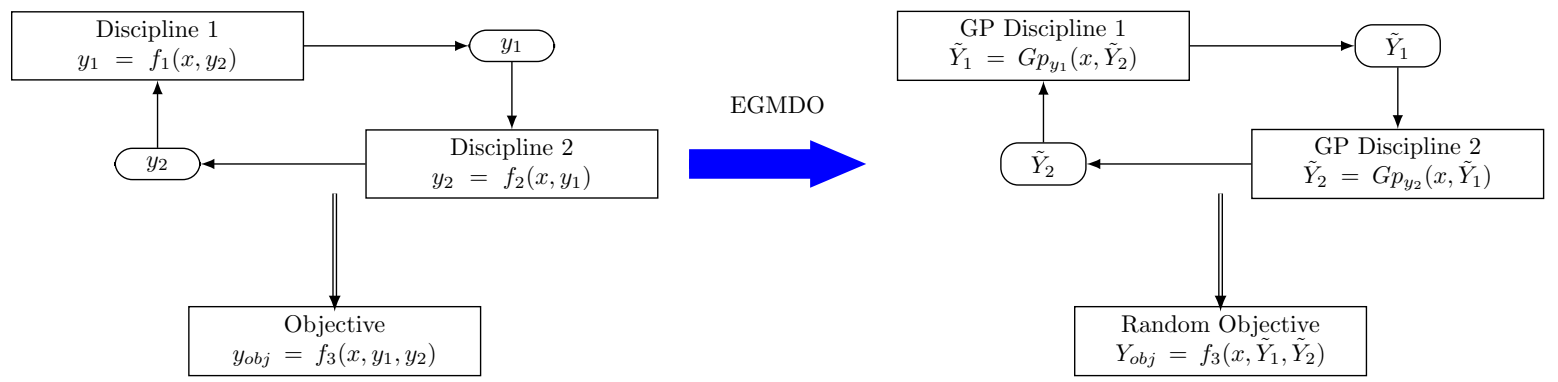

Figure 1. MDO problem involving two coupling disciplines and an objective function. On the left, the disciplines are given by some costly black-boxes. On the right, the disciplines have been replaced by some surrogate models (Gaussian Process denoted by GP).

Among the various MDO formulations proposed in the literature (see 7 for a review), one of the most straightforward to implement, while being quite efficient, is the MultiDisciplinary Feasible (MDF) approach. This method basically consists in optimizing the objective function with respect to the design variable $x$ by solving the MDA at each optimization iteration (leading to feasible solutions at each iteration of the optimizer). Main drawback of this approach is the numerical cost involved in the resolution of the non linear system that must be repeated at every optimization iteration. Hence, in the context of costly disciplinary 
solvers $f_{i}$, MDF approach can lead to intractable computational time. It should be noted that this drawback is amplified in the case of global derivative free optimization problems which is the context of the present study. The direct use of the EGO method ${ }^{1}$ combined with the MDF formulation will therefore imply a large number of disciplinary solver calls.

In order to tackle this issue, the starting point of the proposed approach is to apply the MDF approach on surrogate models of the disciplinary solvers (as presented by the right hand side of Figure 1). Indeed, the numerical cost involved by the resolution of the MDA on surrogate models is negligible. However, in order to be effective, these surrogate models must be accurate near the solution of the optimization problem. Based on previous studies on optimization of black-box functions by surrogate models, one can conclude that adaptive approaches (iterative construction of the surrogate models) are preferable to guarantee accuracy of the solution of the optimization problem at convergence.

In the following, such an adaptive approach is proposed for the particular case of MDO. This approach is inspired by the $\mathrm{EGO}^{1}$ algorithm, however specific difficulties must be tackled. In particular, contrarily to the EGO approach, in the proposed method, it is not the objective function $y_{o b j}$ which is approximated by a Gaussian process but each disciplinary solver $f_{i}$. Hence, the probability distribution of the objective function can not be derived analytically and an uncertainty quantification step has to be performed. Next section details these specific difficulties as well as the specific development we propose in order to tackle them.

\section{B. Description of the main steps}

\section{Gaussian process modelling and random $M D A$}

As explained in the previous part, the basic idea of the proposed approach is to replace each disciplinary solver $f_{i}$ by a surrogate model and to solve the MDA (see Eq. (2)) using these surrogate models. In order to track the uncertainty associated with the use of surrogate models we proposed to use $n_{d}$ independent Gaussian processes to approximate each disciplinary solver. Indeed, based on a given Design of Experiments (DoE), this approach (see 2,8) proposes to model the unknown function as a Gaussian process conditioned on the DoE. Thus, the surrogate model of the unknown function not only provides a prediction of the value of the function but also an uncertainty measure (Gaussian) associated to this prediction. In practice, the disciplinary solver $f_{i}$ is sampled over $\mathcal{X}$ and $C^{(i)}$ (where $C^{(i)}$ denoted the space of the coupling variables for the discipline $i$ ) in order to create an initial DoE, denoted by $\operatorname{DoE}_{f_{i}}$. Consequently, for a given design variable $x^{(0)} \in \mathcal{X}$ and a given coupling variable $y_{c^{(i)}}^{(0)} \in C^{(i)}$ that do not belong to $\operatorname{DoE}_{f_{i}}$, the deterministic solution $y_{i}=f_{i}\left(x^{(0)}, y_{c^{(i)}}^{(0)}\right)$ is approximated by the Gaussian random variable,

$$
\tilde{Y}_{i}\left(x^{(0)}, y_{c^{(i)}}^{(0)}\right)=\mu_{f_{i}}\left(x^{(0)}, y_{c^{(i)}}^{(0)}\right)+\xi_{i}\left(0, \sigma_{f_{i}}\left(x^{(0)}, y_{c^{(i)}}^{(0)}\right)\right)
$$

where $\mu_{f_{i}}\left(x^{(0)}, y_{c^{(i)}}^{(0)}\right)$ is the mean value and $\xi_{i}\left(0, \sigma_{f_{i}}\left(x^{(0)}, y_{c^{(i)}}^{(0)}\right)\right)$ is a Gaussian random variable of zero mean and standard deviation $\sigma_{f_{i}}$ that depends on the position $\left(x^{(0)}\right.$ and $\left.y_{c^{(i)}}^{(0)}\right)$ where the Gaussian process is evaluated. It should be noted that the expressions of $\mu_{f_{i}}$ and $\sigma_{f_{i}}$ are fully specified by the kind of Gaussian process approximation used. For conciseness we choose here not to detail the theory of Gaussian process interpolation and refer the reader to 8 instead. In the following, Gaussian process with constant mean and squared exponential covariance function is used (parameters are classically estimated by maximization of the likelihood function).

The main consequence of using Gaussian processes to approximate each disciplinary solver is that the deterministic non linear system of equations (Eq. (2)) is transformed into a random non linear system of $n_{d}$ equations which reads

$$
\tilde{Y}_{i}\left(x, \tilde{Y}_{c^{(i)}}\right)=\mu_{f_{i}}\left(x, \tilde{Y}_{c^{(i)}}\right)+\xi_{i}\left(0, \sigma_{f_{i}}\left(x, \tilde{Y}_{c^{(i)}}\right)\right), i=1, \cdots, n_{d} \quad \forall x \in \mathcal{X}
$$

whose solution is the joined probability distribution of the random vector of the coupling variables

$\left\{\tilde{Y}_{i}, i=1, \cdots, n_{d}\right\}$. In Eq. (4) the notation $\tilde{Y}_{c^{(i)}}$ stands for the random vector of coupling variables affecting the discipline $i$.

Numerical strategies to approximate the solution of such random non linear system of equations have been studied by several authors (see for example 9, 10, 11). In the following we rely on the method proposed in 11 in which each random response $\tilde{Y}_{i}$ is assumed to be of second order and approximated on an Hermite 
polynomial chaos expansion (see 12,13). This formulation leads to a non linear system of equations where unknowns are the coefficients of each polynomial expansion. In 11 this non linear system of equations is solved by a fixed-point algorithm over the coefficients of the polynomial chaos expansions (at each iteration of the fixed-point algorithm, these coefficients are computed by a classical projection approach, see 11 for details).

Using this approach one obtains an approximation of the solution of the random non linear system of equations Eq. (4) into a polynomial chaos expansion which reads

$$
\hat{Y}_{i}(x, \Xi)=\sum_{j=1}^{P} a_{j}^{(i)}(x) H_{j}(\Xi), i=1, \cdots, n_{d} \quad \forall x \in \mathcal{X}
$$

where $\Xi=\left\{\xi_{i}, i=1, \cdots, n_{d}\right\}$ with $\xi_{i}$ a standard Gaussian random variable, $H_{j}, j=1, \cdots, P$ are the $n_{d}$ variate Hermite polynomials and $P$ is the number of selected polynomial terms. The retained truncation strategy consists in keeping all the polynomials with a degree less or equal to $d$, thus $P=\frac{\left(n_{d}+d\right) !}{n_{d} ! d !}$. Hence, the objective function whose expression in the original deterministic problem reads $y_{o b j}=f_{o b j}\left(x, y_{i}\right)$ is transformed into a random field over the design space $\mathcal{X}$ that can be approximated by a polynomial chaos expansion

$$
\hat{Y}_{o b j}(x, \Xi)=\sum_{j=1}^{P} a_{j}^{(o b j)}(x) H_{j}(\Xi) \quad \forall x \in \mathcal{X} .
$$

It should be noted that the randomness of $\hat{Y}_{o b j}$ translates the uncertainty associated to the use of disciplinary surrogate models. This information is valuable as one looks for the construction of accurate disciplinary surrogate models near the minimum of the objective function. In the following this information will be used to enrich the disciplinary surrogate models in relevant areas of the design space with respect to the search of the deterministic optimum of the objective function.

\section{Remarks:}

- Once the polynomial chaos approximations of the coupling variables are obtained, it is straightforward to compute the polynomial chaos approximation of the objective function (by a non intrusive approach such as projection or regression $\left.{ }^{14}\right)$.

- In theory the accuracy of the polynomial chaos approximation can be increased arbitrary by increasing the degree of the expansion (i.e. the number of polynomial terms $P$ ). However, in practice, increasing the degree of the expansion often leads to numerical stability issues, therefore in the following it is assumed that an accurate polynomial chaos expansion $\hat{Y}_{o b j}$ can be obtained while a low degree polynomial chaos expansion is used (polynomials of degree less or equal to 5).

- It should be noted that polynomial chaos coefficients $a_{j}^{(o b j)}(x), j=1, \cdots, P$ are mapping from $\mathcal{X}$ to $\mathbb{R}$. However they can only be computed pointwise, for a fixed value $x \in \mathcal{X}$. In order to solve this difficulty, next part presents an original approximation of the random field $\hat{Y}_{o b j}(x, \Xi)$ allowing a fast exploration of the design space $\mathcal{X}$.

\section{Representation of the random field $\hat{Y}_{\text {obj }}(x, \Xi)$}

From now on it is assumed that one is able to compute an accurate polynomial chaos expansion of $Y_{o b j}(x)$ for a fixed value of $x$. Let us assume that the uncertainty quantification has been carried out at $n_{U Q}$ points $x^{(k)}, k=1, \cdots, n_{U Q}$ of the design space. Hence, we concatenate the polynomial chaos approximations into a random vector,

$$
\hat{\boldsymbol{Y}}_{o b j}(\Xi)=\left\{\hat{Y}_{o b j}\left(x^{(k)}, \Xi\right), k=1, \cdots, n_{U Q}\right\}
$$

The random vector $\hat{\boldsymbol{Y}}_{o b j}$ is a discretization of the random field $\hat{Y}_{o b j}(x, \Xi)$. This section describes an original continuous approximation of this random field proposed in 15 that will be further used to explore the design space. This continuous representation is based on the Karhunen Loève expansion of the random vector $\hat{\boldsymbol{Y}}_{o b j}$ that can be easily obtained thanks to the coefficients of the polynomial chaos expansions and reads,

$$
\begin{gathered}
\hat{\boldsymbol{Y}}_{o b j}(\Xi)=\boldsymbol{\mu}_{\hat{\boldsymbol{Y}}_{o b j}}+\sum_{k=1}^{n_{U Q}}\left(\sum_{j=2}^{P} \boldsymbol{a}_{j}^{t} \hat{\boldsymbol{\varphi}}_{k} \phi_{j}(\Xi)\right) \hat{\boldsymbol{\varphi}}_{k} \\
4 \text { of } 15
\end{gathered}
$$


where $\boldsymbol{a}_{j}=\left\{a_{j}^{(o b j)}\left(x^{(1)}\right), \cdots, a_{j}^{(o b j)}\left(x^{(k)}\right), \cdots, a_{j}^{(o b j)}\left(x^{\left(n_{U Q}\right)}\right)\right\}, j=2, \cdots, P, \boldsymbol{\mu}_{\hat{\boldsymbol{Y}}_{o b j}}=\boldsymbol{a}_{1}$ and $\hat{\boldsymbol{\varphi}}_{k}$ are the $n_{U Q}$ eigenvectors of the covariance matrix $\boldsymbol{K}_{\hat{\mathbf{Y}}}=\sum_{i=2}^{P} \boldsymbol{a}_{i} \boldsymbol{a}_{i}^{t}$.

From Eq. (7) it is proposed in 15 to approximate the random field $\hat{Y}_{o b j}(x, \Xi)$ by Gaussian process interpolation of the mean value and of the eigenvectors based on the vectors $\boldsymbol{\mu}_{\hat{\boldsymbol{Y}}_{o b j}}$ and $\hat{\boldsymbol{\varphi}}_{j}$ respectively. This leads to the following representation of the random field,

$$
\hat{Y}_{o b j}(x, \Xi) \approx \tilde{Y}(x, \Xi, \boldsymbol{\eta})=\tilde{\mu}_{\hat{\boldsymbol{Y}}_{o b j}}\left(x, \eta_{0}\right)+\sum_{k=1}^{n_{U Q}}\left(\sum_{i=2}^{P} \boldsymbol{a}_{i}^{t} \hat{\boldsymbol{\varphi}}_{k} \phi_{i}(\Xi)\right) \tilde{\varphi}_{k}\left(x, \eta_{k}\right)
$$

where $\tilde{\mu}_{\hat{\boldsymbol{Y}}_{o b j}}\left(x, \eta_{0}\right)$ and $\tilde{\varphi}_{k}\left(x, \eta_{k}\right)$ are respectively the Gaussian process interpolation of the mean vector $\boldsymbol{\mu}_{\hat{\boldsymbol{Y}}_{o b j}}$ and of the eigenvectors $\hat{\boldsymbol{\varphi}}_{k} \cdot \boldsymbol{\eta}=\left[\eta_{0}, \cdots, \eta_{k}, \cdots, \eta_{n_{U Q}}\right]^{t}$ is a random vector of $n_{U Q}+1$ independent normal random variables modeling the uncertainty associated with these Gaussian process interpolations.

\section{Remark:}

- In practice we observed that the random vector $\hat{\boldsymbol{Y}}_{o b j}(\Xi)$ is generally strongly correlated. Hence the Karhunen Loève expansion, given by Eq. (7), can be accurately approximated by truncating it to the $M$ eigenvectors associated to the $M$ highest eigenvalues of the covariance matrix. In practical applications we often observed $M \ll n_{U Q}$ while $M$ is chosen so that the summation of the neglecting eigenvalues is less than $10^{-3 \%}$ of the total eigenvalues sum. This remark is very important in practice as it allows to limit the number of Gaussian process interpolations to construct.

One can note that the approximation of the random field modeling the objective function (Eq. (8)) depends on two sources of uncertainty. The first one, modeled by the random vector $\Xi$, is due to the use of disciplinary Gaussian process surrogate models in the multidisciplinary analysis. The second one, modeled by the random vector $\boldsymbol{\eta}$, is due to the interpolation of the discretization of the random field modelling the random objective function.

It is also notable that Eq. (8) can be evaluated analytically for all points of the design space and for all realizations of the random vectors $\Xi$ and $\boldsymbol{\eta}$. Thus, it is a valuable tool to rapidly explore the objective function with respect to the design variable by considering both sources of uncertainty previously introduced.

Next section presents a criterion that exploits the approximation defined by Eq. (8) in order to find the relevant point of the design space where the accurracy of the disciplinary approximations should be increased, in order for the surrogate based optimum to be more accurate.

\section{Enrichment criterion, uncertainty reduction}

In this section, it is assumed that each disciplinary solver $f_{i}$ has been approximated by a Gaussian process constructed from $\operatorname{DoE}_{f_{i}}$ and that uncertainty quantification by polynomial chaos expansion has been carried out at $n_{U Q}$ points $x^{(k)}, k=1, \cdots, n_{U Q}$; in the following this $\mathrm{DoE}$ is denoted by $\mathrm{DoE}_{U Q}$. Hence an approximation of the random field modelling the objective function (Eq. (8)) is computed. One is now interested in reducing the uncertainty of this random field in the areas of the design space $\mathcal{X}$ where the minimum of the optimization objective function is likely to be.

To do so we first introduce the random variable $\hat{Y}_{m i n}^{(o b j)}(\Xi) \mid x \in \operatorname{DoE}_{U Q}$ which is defined by,

$$
\hat{Y}_{m i n}^{(o b j)}(\Xi) \mid x \in \operatorname{DoE}_{U Q}=\min _{k=1, \cdots, n_{U Q}}\left\{\hat{Y}_{o b j}\left(x^{(k)}, \Xi\right), k=1, \cdots, n_{U Q}\right\}
$$

which is the discretized version of the random variable $\hat{Y}_{m i n}^{(o b j)}(\Xi)$ modelling the minimum value of the random field $\hat{Y}^{(o b j)}(x, \Xi)$ used to approximate the objective function and defined by

$$
\hat{Y}_{\min }^{(o b j)}(\Xi)=\min _{x \in \mathcal{X}} \hat{Y}_{o b j}(x, \Xi)
$$

As our objective is to increase our knowledge on the random variable $\hat{Y}_{\min }^{(o b j)}(\Xi)$ the following Expected Improvement $(E I)$ with respect to both sources of uncertainty previously introduced is defined,

$$
E I(x)=E\left[\left(\hat{Y}_{m i n}^{(o b j)}(\Xi) \mid x \in \operatorname{DoE}_{U Q}-\tilde{Y}(x, \Xi, \boldsymbol{\eta})\right) 1_{\tilde{Y}(x, \Xi, \boldsymbol{\eta}) \leqslant \hat{Y}_{m i n}^{(o b j)}(\Xi) \mid x \in \operatorname{DoE}_{U Q}}\right] \quad \forall x \in \mathcal{X}
$$


where

$1_{\tilde{Y}(x, \Xi, \boldsymbol{\eta}) \leqslant \hat{Y}_{m i n}^{(o b j)}(\Xi) \mid x \in \operatorname{DoE}_{U Q}}=0$ if $\tilde{Y}(x, \Xi, \boldsymbol{\eta})>\hat{Y}_{m i n}^{(o b j)}(\Xi) \mid x \in \operatorname{DoE}_{U Q}$ and

$1_{\tilde{Y}(x, \Xi, \boldsymbol{\eta}) \leqslant \hat{Y}_{m i n}^{(o b j)}(\Xi) \mid x \in \operatorname{DoE}_{U Q}}=1$ if $\tilde{Y}(x, \Xi, \boldsymbol{\eta}) \leqslant \hat{Y}_{m i n}^{(o b j)}(\Xi) \mid x \in \operatorname{DoE}_{U Q}$.

One can note that $E I(x)$ is positive for $x \notin \operatorname{DoE}_{U Q}$ and that $E I(x)=0$ if $x \in \operatorname{DoE}_{U Q}$.

The point $x^{(n e w)}$ where the disciplinary surrogate models should be enriched is thus solution of the optimization problem,

$$
x^{(n e w)}=\arg \max _{x \in \mathcal{X}}(E I(x)) .
$$

It can be noticed that this criterion realizes a compromise between exploitation and exploration. Indeed, at a given point $x^{(0)} \in \mathcal{X}$, a positive value of the Expected Improvement (as defined by Eq. (9)) could have two reasons:

- Large uncertainty due to the Gaussian process interpolation of the design space discretization (modelled by the random vector $\boldsymbol{\eta}$ ) increases the variability of the random variable $\tilde{Y}\left(x^{(0)}, \Xi, \boldsymbol{\eta}\right)$, thus the random event $\left(\hat{Y}_{m i n}^{(o b j)}(\Xi) \mid x \in \operatorname{DoE}_{U Q}>\tilde{Y}\left(x^{(0)}, \Xi, \boldsymbol{\eta}\right)\right)$ may have a non negligible probability. Enrichment in such areas helps the exploration of the design space.

- If the uncertainty associated with the Gaussian Process interpolation of the design space discretization is low (compared to the one due to $\Xi$ ), then a positive value of $E I(x)$ means that the random variable $\tilde{Y}\left(x^{(0)}, \Xi, \boldsymbol{\eta}\right) \approx \hat{Y}_{o b j}\left(x^{(0)}, \Xi\right)$ contributes significantly to the random variable $\hat{Y}_{\min }^{(o b j)}(\Xi)$. This corresponds to the exploitation of the approximation (defined by Eq. (8)), by proposing enrichment of the disciplinary surrogate models.

By considering the expectation over the whole random space ( $\Xi$ and $\boldsymbol{\eta}$ ) the criterion defined by Eq. (9) automatically realizes a compromise between exploration and exploitation.

It should be noted that the $E I$ defined by Eq. (9) is different from the one proposed in 1 in the context of optimization of black-box function. In particular, as the approximation Eq. (8) is not a Gaussian process, the $E I$ defined by Eq. (9) can not be computed analytically and will be estimated by Monte Carlo sampling (see 15 for details about estimation and optimization of the $E I$ ).

After the definition of this criterion, the iterative algorithm we propose simply consists in adding $x^{(n e w)}=$ $\arg \max _{x \in \mathcal{X}}(E I(x))$ to the various disciplinary surrogate models $\operatorname{DoE}_{f_{i}}$ and to the discretization DoE denoted $\operatorname{DoE}_{U Q}$. Then, the disciplinary Gaussian surrogate models are updated and a new approximation Eq. (8) is built in order to evaluate $x^{(n e w)}$. This procedure is repeated until convergence.

Convergence is either defined by a limited number of iterations or by a threshold on the value of $\max _{x \in \mathcal{X}}(E I(x))$. Indeed, once the uncertainty of the approximation of the random field modelling the objective function (given by Eq. (8)) is reduced in the areas of $\mathcal{X}$ where the minimum is likely to be, then $\max _{x \in \mathcal{X}}(E I(x))$ is expected to have a very low value (compared to the one at first iteration for example).

Finally, it is important to notice that the information of $x^{(n e w)}$ is not sufficient to add a point in the disciplinary surrogate $\operatorname{DoE}_{f_{i}}$. Indeed, these surrogate models are constructed over the design space $\mathcal{X}$ and the coupling variables space $C^{(i)}$. In order to find relevant value of these coupling variables at $x^{(n e w)}$, the MDA is solved, at $x^{(n e w)}$ using the mean values of the disciplinary surrogate models. Then, the solution $y_{i}, i=1, \cdots, n_{d}$, is used to add the points $\left(x^{(n e w)}, y_{c^{(i)}}\right)$ to $\operatorname{DoE}_{f_{i}}$.

\section{Exploitation of the obtained random field approximation}

After convergence of the proposed EGMDO algorithm, the following strategy is proposed in order to estimate the distribution of the random variables modeling the minimum value of the objective function and its position. First of all one can note that after convergence, the value of the expected improvement defined by Eq. (9) is expected to be very low, which means that the possible improvement on the random variable $\hat{Y}_{\min }^{(o b j)}(\Xi)$ by adding a new discretization point is negligible. This translates the fact that the random field representation $\tilde{Y}(x, \Xi, \boldsymbol{\eta})$ reaches an acceptable accuracy in the areas of $\mathcal{X}$ where the minimum is likely to be and consequently that uncertainty due to the Gaussian process interpolation (modeled by the $\boldsymbol{\eta}$ random variable) is negligible. Hence, we propose to use the mean value of the Gaussian process interpolation in the 
random field representation given by Eq. (8) leading to

$$
\hat{Y}_{o b j}(x, \Xi) \approx \tilde{Y}(x, \Xi)=\mu_{\tilde{\mu}_{\hat{\Upsilon}_{o b j}}}(x)+\sum_{k=1}^{n_{U Q}}\left(\sum_{i=2}^{P} \boldsymbol{a}_{i}^{t} \hat{\boldsymbol{\varphi}}_{k} \phi_{i}(\Xi)\right) \mu_{\tilde{\varphi}_{k}}(x)
$$

where $\mu_{\tilde{\mu}_{\hat{\varphi}_{o b j}}}(x)$ and $\mu_{\tilde{\varphi}_{k}}(x)$ are respectively the mean value of the Gaussian process interpolation of the mean vector $\boldsymbol{\mu}_{\hat{\boldsymbol{Y}}_{o b j}}$ and of the eigenvectors $\hat{\boldsymbol{\varphi}}_{k}$.

Then, Eq. (11) can be seen as a metamodel of the random field modelling the objective function optimized to be accurate in the areas of $\mathcal{X}$ where the minimum is likely to be. As a consequence it will be used in the following to estimate the probability distribution of the random variables modeling the minimum value of the objective function and its position such as,

$$
\hat{Y}_{\min }^{(o b j)}(\Xi)=\min _{x \in \mathcal{X}}\left(\hat{Y}_{o b j}(x, \Xi)\right) \approx \min _{x \in \mathcal{X}}\left(\tilde{Y}_{o b j}(x, \Xi)\right)
$$

and

$$
\hat{X}^{\star}(\Xi)=\arg \min _{x \in \mathcal{X}}\left(\hat{Y}_{o b j}(x, \Xi)\right) \approx \min _{x \in \mathcal{X}}\left(\tilde{Y}_{o b j}(x, \Xi)\right)
$$

Moreover, it should be noted that the random field representation given by Eq. (11) only involves polynomial functions and thus makes the estimation of $\hat{Y}_{\min }^{(o b j)}(\Xi)$ and $\hat{X}^{\star}(\Xi)$ easily accessible by the Monte Carlo method (i.e. solving the minimization problem of Eq. (12) for a large number of samples $\Xi^{(j)}, j=1, \cdots, n_{\text {sim }}$ ).

Next section presents two applications of this strategy on an academic test case proposed in 4 and on a more realistic aircraft design test case in the context of the AGILE European project. ${ }^{5,6}$

\section{Applications}

\section{A. The analytical Sellar problem}

The analytic test case proposed in 4 is defined by the following set of equations:

$$
\begin{aligned}
& \min _{x_{1}, x_{2}, x_{3}} y_{o b j}\left(x_{1}, x_{2}, x_{3}, y_{1}, y_{2}\right)=x_{3}^{2}+x_{1}+y_{1}+\exp -y_{2} \\
& \text { subject to: } \\
& \frac{y_{1}}{3.16}-1 \geqslant 0 \\
& 1-\frac{y_{2}}{24} \geqslant 0 \\
& x_{1} \in \mathcal{X}_{1}=[-10,10] \\
& x_{2} \in \mathcal{X}_{2}=[0,10] \\
& x_{3} \in \mathcal{X}_{3}=[0,10] \\
& \text { where : } \\
& y_{1}=f_{1}\left(x_{1}, x_{2}, x_{3}, y_{2}\right)=x_{1}^{2}+x_{3}+x_{2}-0.2 y_{2} \\
& y_{2}=f_{2}\left(x_{1}, x_{2}, y_{1}\right)=\sqrt{y_{1}}+x_{1}+x_{2} .
\end{aligned}
$$

The ranges for the design space and the coupling variables are given by

$$
\begin{gathered}
\mathcal{X}=\mathcal{X}_{1} \times \mathcal{X}_{2} \times \mathcal{X}_{3}=[-10,10] \times[0,10] \times[0,10] \\
C^{(1)}=[-5.0,24], \quad C^{(2)}=[1.0,50] .
\end{gathered}
$$

Note that even though all expressions are analytical we consider the test case as representative of an MDO problem involving expensive black box disciplinary functions, thus considering that we do not know the true disciplinary functions but can only evaluate them at discrete points of the design space. Accordingly, two independent Gaussian process surrogate models are built in order to replace the two disciplinary functions $f_{1}$ and $f_{2}$. In order to construct the Gaussian process surrogate model of $f_{1}$, the $\operatorname{DoE}_{f_{1}}$ is constructed on the design and coupling variables space of the first discipline, namely $\mathcal{X}_{1} \times \mathcal{X}_{2} \times \mathcal{X}_{3} \times C^{(1)}$. It should be noted that one of the difficulties of the proposed approach is the definition of the variation domain of the coupling variable (in that case $y_{2}$ ). In practice, this definition should be provided by an expert of the discipline. In this simple case, after investigation we simply propose $C^{(1)}=[-5.0,24.0]$. We recall that the idea is to 
iteratively improve the surrogate model in the interesting area of the coupling and design variables space, thus the initial $\mathrm{DoE}_{f_{1}}$ is constructed by LHS sampling of only 5 points. Concerning the second discipline, $f_{2}$, the initial DoE is also constructed by LHS and counts 5 points. DoE $f_{2}$ is sampled over the space of dimension $3, \mathcal{X}_{1} \times \mathcal{X}_{2} \times C^{(2)}$ where $C^{(2)}$ is the variation domain of $y_{1}$ and it is set to $C^{(2)}=[1.0,50.0]$ in that example. Following the idea presented in section II.B, the objective function is now considered as a random field over the design variable space $\mathcal{X}$. This random field is discretized on a $\mathrm{DoE}_{U Q}$ of 10 points sampled by LHS over $\mathcal{X}$. Consequently, the minimum value of the objective function is a random variable. This random variable can be easily evaluated by Monte Carlo sampling thanks to the approximation Eq. (11) described in section II.B.4 (see 15 for details). Figure 2 presents a histogram (constructed with $n_{\text {sim }}=500$ ) of this random variable at the initial step (we recall that both disciplines are approximated by independent Gaussian process surrogate models built with only 5 points).

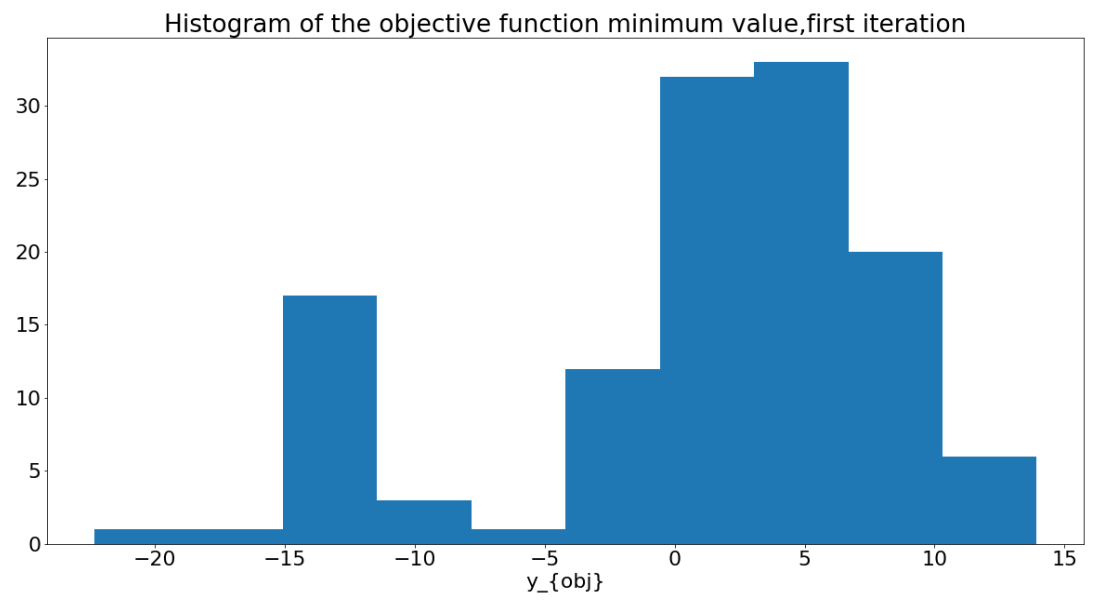

Figure 2. Histogram of the objective function minimum value at the initial step (500 random realizations).

One can note that the dispersion of this random variable is very large (mean $\approx 0.94$, coefficient of variation $\approx 790 \%$ ). This large dispersion is explained by the fact that the surrogate models of the disciplines are not accurate at all in the area where the minimum of the objective function can be reached (which is expected as they are constructed with only 5 points sampled over the whole design and coupling variables space).

Objective of the proposed method is now to enrich the surrogate model until the random variable modelling the minimum value of the objective function reaches a sufficiently small dispersion. Application of the proposed algorithm leads to a convergence after 7 iterations $\left(7\right.$ points are added to the disciplinary $\operatorname{DoE}_{f_{i}}$ and to the discretization $\operatorname{DoE}_{U Q}$ ). Figure 3 presents the dispersion of the random variable modelling the minimum value of the objective function after convergence of the proposed method (i.e. the histogram is estimated by the method described in section II.B.4 with $n_{s i m}=500$ ). Note that by solving the optimization problem $n_{\text {sim }}$ times, as described section II.B.4, we obtain samples for both the value of the minimum $y_{o b j}^{\star}$ and for its position $x^{\star}$.

Figure 3 shows that the proposed iterative approach leads to a strong reduction of the uncertainty on the minimum value of the objective function (mean $\approx 3.1847$, coefficient of variation $\approx 0.3 \%$ ). Moreover, one can note that the minimum value is in agreement with the reference result $(\approx 3.18339)$.

Table 1 presents comparisons done between an MDF formulation with a gradient optimizer (SLSQP ${ }^{16}$ ) or a gradient free optimizer $\left(\mathrm{COBYLA}^{17}\right)$ and EGMDO approach in terms of values of optimal design variables and objective function (mean value and coefficient of variation associated to the EGMDO optimum and its position) and also the number of calls for each disciplinary solver. Note that for the MDF formulation with the gradient based algorithm, the number of calls includes the computation of the objective function and its derivative by finite-differences.

Results of Table 1 shows that EGMDO approach converges towards the same optimum as MDF approaches by only evaluating 12 times the disciplinary solvers (compared to 532 and 443 for MDF approaches). This large reduction is explained by the intensive use of disciplinary surrogate models in the EGMDO strategy instead of actual disciplinary solvers in MDF strategies. Note however, that the numerical cost of the EGMDO strategy should also include the creation of the disciplinary surrogate models, the propagation of 


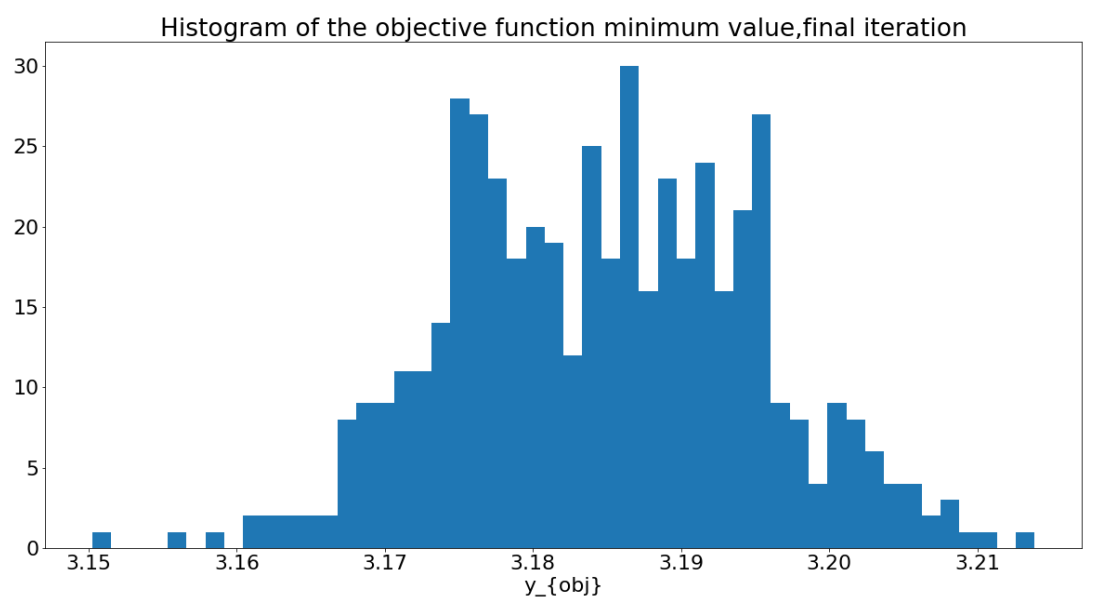

Figure 3. Histogram of the minimum value of the objective function after convergence of the proposed method (500 random realizations).

\begin{tabular}{c|c|c|c} 
& MDF SLSQP & MDF COBYLA & EGMDO \\
\hline \hline$y_{o b j}^{\star}$ & 3.1834 & 3.1835 & $3.1847(0.32 \%)$ \\
$x_{1}^{\star}$ & 1.977 & 1.977 & $1.977\left(10^{-11 \%)}\right.$ \\
$x_{2}^{\star}$ & 0.0 & 0.0 & $10^{-14}\left(10^{-4} \%\right)$ \\
$x_{3}^{\star}$ & 0.0 & 0.0 & $10^{-17}\left(10^{-4} \%\right)$ \\
\hline \# calls of each discipline & 532 & 443 & $12(5+7)$
\end{tabular}

Table 1. Comparisons between a MDF formulation solved with SLSQP or COBYLA (with computation of disciplinary gradient by finite differences for SLSQP) and the EGMDO approach on the Sellar problem. For the EGMDO approach, the mean value of each variable if given and the coefficient of variation is mentioned in parenthesis.

uncertainty by PCE, and the optimization of the enrichment criterion Eq. (10). These steps only involve surrogate models and their numerical cost depends in large on the numerical implementation of the EGMDO strategy. We found that these steps were negligible on the expensive disciplinary models we considered but this will vary from problem to problem. Note also that many parts of EGMDO algorithm can be easily parallelized in order to increase the efficiency of the overhead computations.

Concerning the position of the minimum in the design and coupling variables space, Figure 4 presents the points added by the proposed algorithm to $\mathrm{DoE}_{f_{1}}$ and $\mathrm{DoE}_{f_{2}}$.

First, one can see on Figure 4 that the initial DoE (green lines) is well spread over the whole design and coupling variables space for both disciplines. Then, the blue lines show the points added by the proposed method. One can note that after approximately 4 iterations of domain exploration, the interesting area is found by the proposed algorithm and that convergence to the reference result (red dotted curve) is achieved in few more iterations.

Finally we access the global character of EGMDO algorithm by using 10 random initial DOE of size 5 . EGMDO algorithm converges towards the global optimum 10 times over the 10 random initial DOE with a mean number of iterations equals to 7.7 (better 6, worst 12). It should be noted that MDF formulation with either SLSQP or COBYLA optimizer performed a local search and it is consequently very dependent of the starting point, using 10 different random starting points both MDF approaches converge only 6 times towards the global optimum. So in terms of robustness on the Sellar problem, EGMDO is much more efficient with a higher success rate (global optimum found 10 times out of 10 runs, $100 \%$ of success rate) than an MDF approach (global optimum found 6 times out of 10 runs, $60 \%$ of success rate).

As a conclusion, this example illustrates the interest of a global MDO approach by an enrichment procedure. Indeed, by carefully considering the uncertainty associated to the use of Gaussian process surrogate model, the proposed approach aims at reducing the uncertainty on the minimum value of the objective function until an acceptable uncertainty remains. This approach allows a strong reduction in the number 


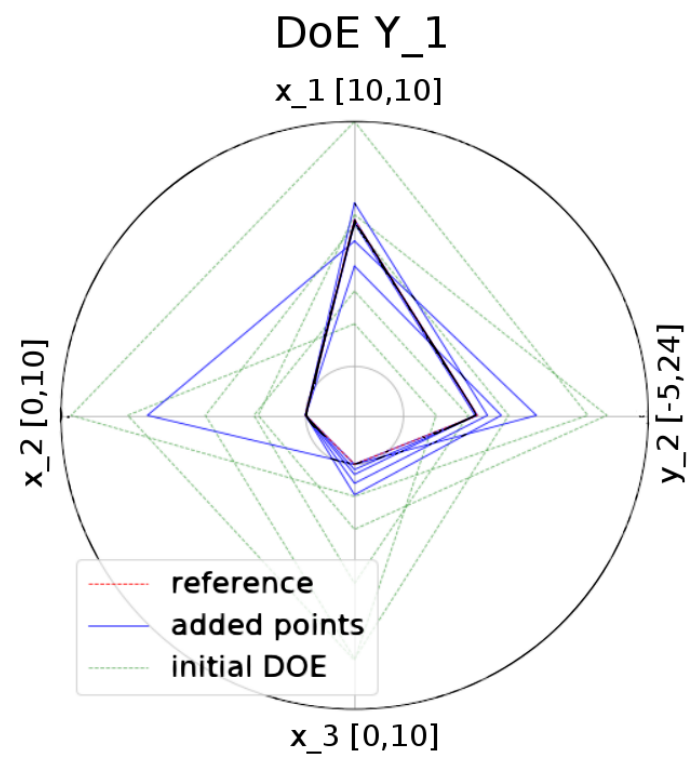

(a) $Y_{1}$ coupling variable

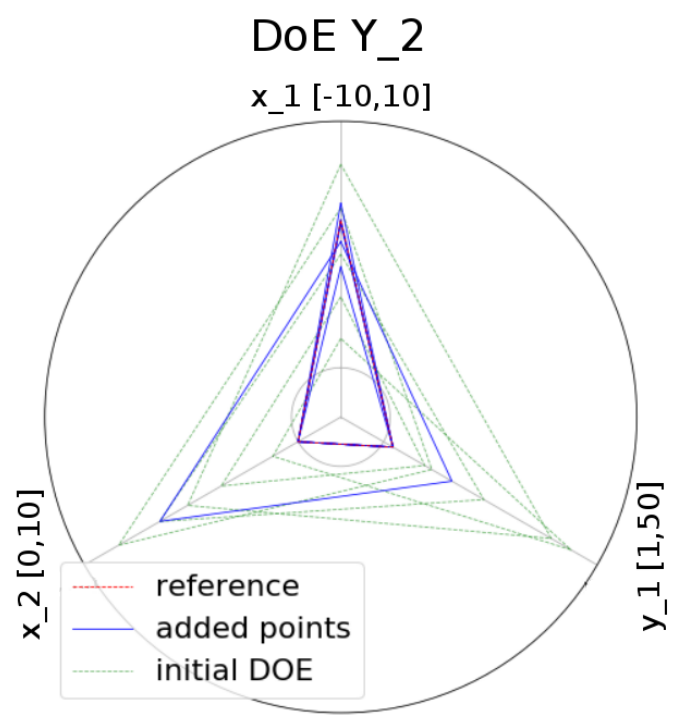

(b) $Y_{2}$ coupling variable

Figure 4. Evolution of the design and coupling variables DoE for the adaptive construction of disciplinary surrogate models. The initial DoE is represented by the green lines, the added points are in blue and the reference solution is in red.

of evaluations of the disciplinary solvers but induces an additional numerical cost for setting up the strategy (construction of disciplinary surrogate models, propagation of uncertainty by PCE, optimization of the enrichment criterion).

\section{B. The AGILE aircraft design}

This test case is developed in the framework of the H2020 European project AGILE ${ }^{18}$ (Aircraft third Generation MDO for Innovative Collaboration of Heterogeneous Teams of Experts) and concerns the MDO of a civil aircraft. The test case consists of the design and optimization task for a large regional jet, with Entry Into Service 2020. Starting from the specification of the Top Level Aircraft Requirements provided by the aircraft manufacturer partner (Bombardier), an Overall Aircraft Design (OAD) task targeting conceptual and preliminary development stages was implemented during the project. Related to the use case, a specific scenario was considered, related to the investigations of MDO formulations on complex workflows, representative of an aircraft design process. For that aim, clusters of disciplinary solvers used in the MDA of the large regional jet were built to derive specific solvers to be used in the scenario.

On-board systems Solver This solver aims at providing the On Board systems performance, in terms of weights and power, using the wing design parameters and other inputs such as the Fuel Weight and other operational weights such as MTOM (Maximum Take-Off Mass).

Structural sizing and Weight Solver This solver provides the OEM (Operating Empty Mass) of the whole aircraft, using as inputs the wing design parameters, the fuel weight and the systems weight. It also contains the load and structural sizing competence that sizes the wing structure and computes its weight.

Weight Update Solver This solver provides the update of the operational weights of the whole aircraft, using as inputs the fuel weights and the systems weight.

Mission performance Solver This solver provides the Mission performance calculation and uses as inputs, the wing design parameters, the operational weights and the Aerodynamic look up tables to run the full mission and provides the fuel weight. 
Eventually, Figure 5 presents both the reference aircraft configuration and the MDA workflows obtained with 4 disciplinary solvers defined above, representative of an aircraft design problem with different disciplines (Aerodynamic, Structure, Performance) coupled together (more information on the scenario can be found in 19).

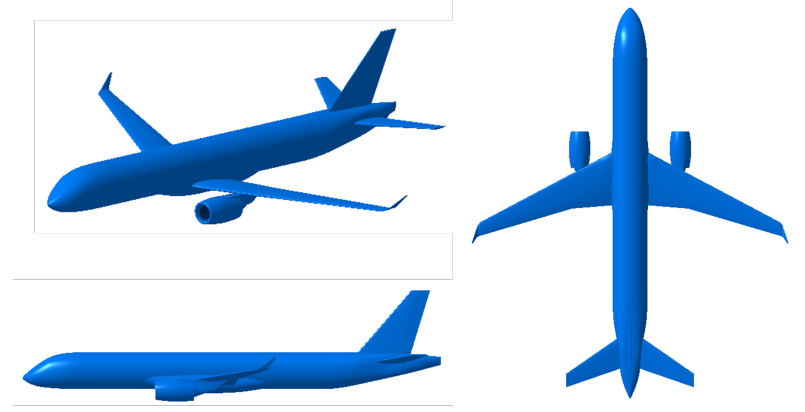

(a) AGILE regional jet configuration

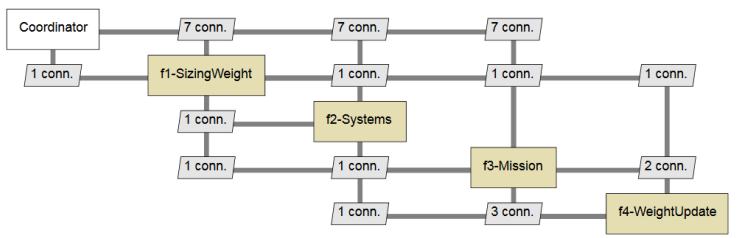

(b) Workflow of MDA with the 4 disciplinary solvers

Figure 5. AGILE project test case.

This MDA is characterized by 7 coupling variables and 7 design variables. The multidisciplinary analysis is defined by the following non linear system of equations

$$
\begin{array}{ll}
m_{O E M} & =f_{1}\left(x, m_{\text {Systems }}, m_{F M}, m_{T O M}\right) \\
m_{\text {Systems }} & =f_{2}\left(x, m_{O E M}, m_{T O M}, m_{F M}\right) \\
m_{F u e l}, m_{F M} & =f_{3}\left(x, m_{O E M}, m_{T O M}, m_{Z F M}, m_{M L M}\right) \\
m_{T O M}, m_{Z F M}, m_{M L M} & =f_{4}\left(m_{O E M}, m_{F M}, m_{F u e l}\right)
\end{array}
$$

where $m_{O E M}, m_{\text {Systems }}, m_{F u e l}, m_{F M}, m_{T O M}, m_{Z F M}, m_{M L M}$ are the coupling variables and $x=$ $\left\{t_{k}, t_{t}, t c_{k}, t c_{t}, w_{A}, w_{S}, a r\right\}$ is the vector of design variables that define the wing shape. Table 2 presents these 14 variables. Solvers $f_{i}, i=1, \cdots, 4$ are clusters of disciplinary solvers provided by various members of the AGILE project. It should be noted that these clusters are simplified version of high fidelity numerical solvers. Indeed, purpose of this test case is to easily test various MDO architectures in a realistic aircraft design context while maintaining reasonable numerical cost in order to easily access reference results by standard approach (MDF). In order to evaluate the capability of the EGMDO algorithm the following unconstrained minimization problem is set up:

$$
\min _{x \in \mathcal{X}} m_{O E M}
$$

such as $m_{O E M}$ is solution of Eq. (13).

Reference results are obtained by an MDF approach with SLSQP algorithm and COBYLA algorithm (as for the previous example jacobian, is evaluated by finite differences within the SLSQP algorithm). Table 3 presents the results obtained and the number of disciplinary solvers evaluations. It should be noted that the value of the objective function for $x^{0}$ located at the middle of the design space is $m_{O E M}\left(x^{0}\right) \approx 30073$.

One can note on Table 3 that both MDF approaches converge to almost the same minimum.

In order to setup the EGMDO approach, Gaussian process surrogate models of disciplinary solvers $f_{1}$, $f_{2}$ and $f_{4}$ are build based on three independent DoE of size 30. The Gaussian process surrogate model of disciplinary solver $f_{3}$ is build on a DoE of size 50 (this one counts more coupling variables and higher nonlinearity is observed).

The initial $\mathrm{DoE}_{U Q}$ used for the discretization of the random field modeling the objective function counts 30 points. EGMDO algorithm converges after 9 iterations. Figure 6 shows an histogram of the objective function minimum value after convergence of the EGMDO algorithm obtained by solving 500 optimization problems using the final surrogate model of the random field modeling the objective function (Eq. (11)). As for the previous example one can see that at convergence, the minimum value of the objective function almost reached a Dirac distribution. Table 4 presents the results in terms of variable values obtained at convergence with the mean value and the coefficient of variation estimated from the 500 random realizations. The relative error (denoted by $\epsilon$ ) between these mean values and the reference ones obtained by MDF-SLSQP approach is 


\begin{tabular}{c|c|c|c}
\hline \multicolumn{4}{|c}{ Design variables } \\
\hline \hline name & designation & $\min$ & $\max$ \\
\hline$t_{k}$ & twist kink & -3 & 3 \\
$t_{t}$ & twist Tip & -5 & 2 \\
$t c_{k}$ & thickness over chord ratio kink & 0.10 & 0.12 \\
$t c_{t}$ & thickness over chord ratio tip & 0.09 & 0.11 \\
$w_{A}$ & wing area & 75.0 & 95.0 \\
$w_{S}$ & wing sweep & 30.0 & 34.0 \\
$a r$ & aspect ratio & 9.0 & 10.5 \\
\hline \multicolumn{4}{|c}{ Coupling variables } \\
\hline \hline name & designation & $\min$ \\
\hline$m_{O E M}$ & Operating Empty Mass & 27000 & 28500 \\
$m_{\text {Systems }}$ & Systems Mass & 7800.0 & 8400.0 \\
$m_{F u e l}$ & Mission Fuel Mass & 5000 & 6500 \\
$m_{F M}$ & Total Fuel Mass & 6800 & 7600 \\
$m_{T O M}$ & Maximum Take Off Mass & 43000 & 46000 \\
$m_{Z F M}$ & Zero Fuel Mass & 35200 & 37500 \\
$m_{M L M}$ & Maximum Landing Mass & 36500 & 39100 \\
\hline
\end{tabular}

Table 2. Description of the design variables and coupling variables of the problem. Note that for the coupling variables the given ranges of variation are initial guesses based on an expert judgment and that coupling variables might take values outside these ranges during the MDA resolution.

\begin{tabular}{ccccccccc|c} 
& $t_{k}$ & $t_{t}$ & $t c_{k}$ & $t c_{t}$ & $w_{A}$ & $w_{S}$ & ar & $m_{O E M}$ & \# calls of each discipline \\
\hline MDF-SLSQP & -3 & -5 & 0.1012 & 0.09352 & 75 & 30 & 9 & 29193.107 & 1149 \\
MDF-COBYLA & -3 & -5 & 0.1009 & 0.09346 & 75 & 30 & 9 & 29193.110 & 1057
\end{tabular}

Table 3. Reference results for the AGILE test case obtained using an MDF based on SLSQP or COBYLA algorithm. 
also reported. One can note that the EGMDO approach leads to an optimal point close to the reference one (the worst relative error is for the design variable $t c_{t}$ and equals $3.76 \%$ ) and to an optimal value in agreement with the reference. Moreover, computing the MDA with the actual disciplinary solver at the optimal point found by the EGMDO algorithm leads to $m_{O E M}=29196.777$ which validates the approximation constructed by EGMDO, of the objective function near the optimal point of the design space.

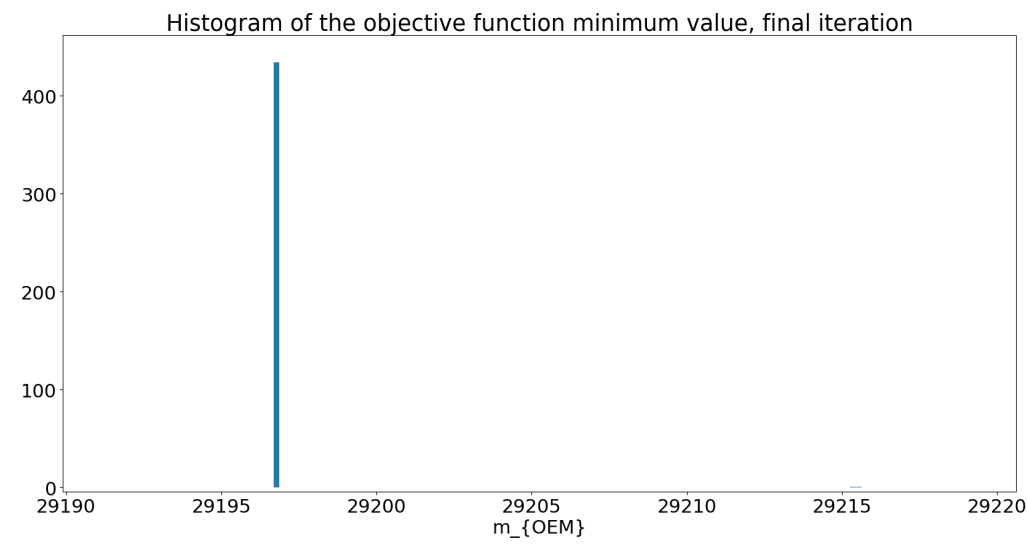

Figure 6. AGILE example: Histogram of the minimum value of the objective function after convergence of the proposed method (500 random realizations).

\begin{tabular}{ccccccccc} 
& $t_{k}$ & $t_{t}$ & $t c_{k}$ & $t c_{t}$ & $w_{A}$ & $w_{S}$ & $a r$ & $m_{O E M}$ \\
\hline EGMDO & $-3\left(10^{-10 \%)}\right.$ & $-5(0 \%)$ & $0.1034(1.08 \%)$ & $0.09(0 \%)$ & $75(0 \%)$ & $30(0 \%)$ & $9(0 \%)$ & $29196.852\left(4.10^{-3} \%\right)$ \\
$\epsilon$ & 0 & 0 & 0.0217 & 0.0376 & 0 & 0 & 0 & $10^{-4}$
\end{tabular}

Table 4. Results obtained with the EGMDO approach, the mean value of each variable is given and the coefficient of variation is mentioned in parenthesis. $\epsilon$ stands for the relative error compared to the results obtained with MDF-SLSQP approach.

Finally one can conclude on this example that, by only using a few calls to the actual disciplinary solvers (39 for solvers $f_{1}, f_{2}$ and $f_{4}$ ) and (59 for the solver $f_{3}$ ), the proposed EGMDO approach allows to identify the area of the design space where the deterministic optimum is likely to be (compared to 1149 calls for the reference MDF-SLSQP approach). Consequently, EGMDO approach can be used either in MDO context in which the number of disciplinary solvers evaluation is extremely limited or in order to rapidly finds relevant starting point for local MDO approach (MDF-SLSQP for example). This last point is not developed in the present article but will be investigated in future development.

\section{Conclusion and perspectives}

A multidisciplinary optimization problem has been solved using a proposed surrogate based approach. Each disciplinary solver is replaced by a surrogate model (a Gaussian process) allowing to use the mean value and the associated modelling uncertainty in the search of the optimum. This is achieved by propagating the uncertainties introduced by the use of the surrogate models to the objective function, followed by an iterative process seeking to enrich the surrogate models by calling the disciplinary solvers at some well chosen points in order to increase their accuracy in the vicinity of the optimum. This iterative process tends to reduce the uncertainty in the optimum of the objective function and to converge toward the deterministic minimum of the initial problem.

This strategy mainly relies on the development of a new random field approximation based on polynomial chaos expansion and Gaussian process interpolation proposed in 15. Indeed, this approximation eases the exploration of the design space by taking into account the variability of the random field (due to the modeling uncertainty on the disciplnary surrogates and modelled by the random vector $\Xi$ ) and the interpolation uncertainty (due to the discretization of the random field and modelled by the random vector $\boldsymbol{\eta}$ ). In order 
to efficiently take advantage of this approximation an adaptation of the expected improvement criterion is proposed.

The proposed EGMDO strategy has been validated on an analytic MDO test case: the Sellar problem, ${ }^{4}$ involving two disciplines with two coupling variables. A larger experimentation has been done within the H2020 AGILE project ${ }^{5}$ where 7 design variables and 7 coupling variables were involved for an aircraft design test case. These two applications gave very promising results in terms of number of function evaluations to find the optimal value of the MDO problem: a reduction by a factor of 30 is obtained by comparison to an MDF-SLSQP approach.

Finally, the advantages of the EGMDO method can be summarized as:

- Fully uncoupled approach: each disciplinary surrogate model is built independently.

- Numerical efficiency: the MDA is never solved using the disciplinary solvers. This step is replaced by an uncertainty quantification process involving only the surrogate models.

- The number of calls to the disciplinary solver is reduced by carefully selecting the discretization points used to build the surrogate models. The surrogate models are thus only accurate in the area of the design and coupling variables space where the minimum is likely to be. We recall that the previous results on the Sellar example are obtained by only 12 calls to the disciplinary solvers and for the aircraft test case 39 or 59 calls (depending on the solver) are necessary.

- As the probabilistic framework is used, the final results (minimum value and position of the minimum) are random variables, which allow to easily quantifying the confidence on these results.

Some points such as the number of coupling variables and their domain definition are still under study. Indeed, increasing the number of coupling variables increases the stochastic dimension of the problem which makes it difficult to solve with our current implementation of polynomial chaos expansion. Some solutions to deal with larger dimension will be explored in future work.

\section{Acknowledgments}

The research presented in this paper has been performed in the framework of the AGILE project (Aircraft 3rd Generation MDO for Innovative Collaboration of Heterogeneous Teams of Experts) and has received funding from the European Union Horizon 2020 Programme (H2020-MG-2014-2015) under grant agreement $\mathrm{n}^{\mathrm{o}}$ 636202. The authors are grateful to the partners of the AGILE consortium for their contribution and feedback, especially to our colleagues from DLR, UNINA, POLITO and NOESIS who provided the AGILE use case's models.

\section{References}

${ }^{1}$ Jones, D. R., Schonlau, M., and Welch, W. J., "Efficient Global Optimization of Expensive Black-Box Functions," Journal of Global Optimization, Vol. 13, No. 4, 1998, pp. 455-492.

${ }^{2}$ Forrester, A., Sobester, A., and Keane, A., Engineering design via surrogate modelling: a practical guide, John Wiley \& Sons, Hoboken, NJ, 2008.

${ }^{3}$ Sasena, M. J., Flexibility and efficiency enhancements for constrained global design optimization with kriging approximations, Ph.D. thesis, University of Michigan, 2002.

${ }^{4}$ Sellar, R. S., Batill, S. M., and Renaud, J. E., "Response Surface Based, Concurrent Subspace Optimization For Multidisciplinary System Design," 34th AIAA Aerospace Sciences Meeting and Exhibit, No. 96-0714, 1996, pp. 96-0714.

5 "AGILE EU Project portal," http://www.agile-project.eu.

${ }^{6}$ Ciampa, P. D. and Nagel, B., "Towards the 3rd generation MDO collaboration Environment," 30th ICAS Congress, Daejeon, Korea, September 2016.

${ }^{7}$ Chittick, I. R. and Martins, J. R. R. A., "An asymmetric suboptimization approach to aerostructural optimization," Optimization and Engineering, Vol. 10, No. 1, 2008, pp. 133-152.

${ }^{8}$ Rasmussen, C. E. and Williams, C. K. I., Gaussian processes for machine learning, Adaptive computation and machine learning, MIT Press, Cambridge, Mass, 2006.

${ }^{9}$ Arnst, M., Ghanem, R., Phipps, E., and Red-Horse, J., "Dimension reduction in stochastic modeling of coupled problems," International Journal for Numerical Methods in Engineering, Vol. 92, No. 11, 2012, pp. 940-968.

${ }^{10}$ Sankararaman, S. and Mahadevan, S., "Likelihood-Based Approach to Multidisciplinary Analysis Under Uncertainty," Journal of Mechanical Design, Vol. 134, No. 3, 2012, pp. 03100812 pages.

${ }^{11}$ Dubreuil, S., Bartoli, N., Gogu, C., and Lefebvre, T., "Propagation of Modeling uncertainty by Polynomial Chaos Expansion in Muldisciplinary Analysis," Journal of Mechanical Design, Vol. 138, No. 11, 2016, pp. 111411. 
${ }^{12}$ Cameron, R. H. and Martin, W. T., "The Orthogonal Development of Non-Linear Functionals in Series of Fourier-Hermite Functionals," Annals of Mathematics, Vol. 48, No. 2, 1947, pp. 385-392.

${ }^{13}$ Ghanem, R. G. and Spanos, P. D., Stochastic finite elements: a spectral approach, Springer, New York, NY, 1991.

${ }^{14}$ Berveiller, M., Sudret, B., and Lemaire, M., "Stochastic finite elements: a non-intrusive approach by regression," European Journal of Computational Mechanics, Vol. 15, No. 1-3, 2006, pp. $81-92$.

${ }^{15}$ Dubreuil, S., Bartoli, N., Gogu, C., Lefebvre, T., and Colomer, J. M., "Extreme value oriented random field discretization based on an hybrid polynomial chaos expansion-Kriging approach," Computer Methods in Applied Mechanics and Engineering, 2018.

${ }^{16} \mathrm{Kraft}$, D. et al., A software package for sequential quadratic programming, DFVLR Obersfaffeuhofen, Germany, 1988.

${ }^{17}$ Powell, M. J. D., "An efficient method for finding the minimum of a function of several variables without calculating derivatives," The Computer Journal, Vol. 7, No. 2, 1964, pp. 155-162.

${ }^{18}$ Ciampa, P. D. and Nagel, B., "The AGILE Paradigm: the next generation of collaborative MDO," 18th AIAA/ISSMO Multidisciplinary Analysis and Optimization Conference, Denver, USA, June 2017.

${ }^{19}$ Lefebvre, T., Bartoli, N., Dubreuil, S., Panzeri, M., Lombardi, R., Lammen, W., Zhang, M., van Gent, I., and Ciampa, P. D., "Overview Of MDO Enhancement In The AGILE Project: A Clustered And Surrogate-Based MDA Use Case," 6th CEAS Aerospace Europe Conference, October 2017. 